\title{
A New Power Sharing Scheme of Multiple Microgrids and an Iterative Pairing-Based Scheduling Method
}

\author{
Hong-Chao Gao ${ }^{1}$, Joon-Ho Choi ${ }^{2} \mathbb{D}$, Sang-Yun Yun ${ }^{2} \mathbb{D}$ and Seon-Ju Ahn ${ }^{2, *}$ \\ 1 State Key Lab of Power Systems, Department of Electrical Engineering, Tsinghua University, Beijing 100084, \\ China; hcgao@tsinghua.edu.cn \\ 2 Department of Electrical Engineering, Chonnam National University, 77, Yongbong-ro, Buk-gu, \\ Gwangju 61186, Korea; joono@chonnam.ac.kr (J.-H.C.); drk9034@jnu.ac.kr (S.-Y.Y.) \\ * Correspondence: sjahn@jnu.ac.kr; Tel.: +82-62-530-1738
}

Received: 25 February 2020; Accepted: 30 March 2020; Published: 1 April 2020

\begin{abstract}
As the numbers of microgrids (MGs) and prosumers are increasing, many research efforts are proposing various power sharing schemes for multiple MGs (MMGs). Power sharing between MMGs can reduce the investment and operating costs of MGs. However, since MGs exchange power through distribution lines, this may have an adverse effect on the utility, such as an increase in peak demand, and cause local overcurrent issues. Therefore, this paper proposes a power sharing scheme that is beneficial to both MGs and the utility. This research assumes that in an MG, the energy storage system (ESS) is the major controllable resource. In the proposed power sharing scheme, an MG that sends power should discharge at least as much power from the ESS as the power it sends to other MGs, in order to actually decrease the total system demand. With these assumptions, methods for determining the power sharing schedule are proposed. Firstly, a mixed integer linear programming (MILP)-based centralized approach is proposed. Although this can provide the optimal power sharing solution, in practice, this method is very difficult to apply, due to the large calculation burden. To overcome the significant calculation burden of the centralized optimization method, a new method for determining the power sharing schedule is proposed. In this approach, the amount of power sharing is assumed to be a multiple of a unit amount, and the final power sharing schedule is determined by iteratively finding the best MG pair that exchange this unit amount. Simulation with a five MG scenario is used to test the proposed power sharing scheme and the scheduling algorithm in terms of a reduction in the operating cost of MGs, the peak demand of utility, and the calculation burden. In addition, the interrelationship between power sharing and the system loss is analyzed when MGs exchange power through the utility network.
\end{abstract}

Keywords: microgrid; multiple microgrids; power sharing schedule; energy storage system; microgrid aggregator

\section{Introduction}

In recent years, as the capacity of renewables integrated into the grid has continued to expand, the construction of microgrids (MGs) has gradually increased [1]. To improve the energy efficiency and to ensure the economical operation of MGs, energy management technologies are experiencing rapid innovation. The energy storage system (ESS) is one of the key components for microgrid control, and thus optimal scheduling of the ESS is very important for improving the operational performance of a microgrid [2]. Therefore, increasing the ESS capacity can make the MG operation more efficient and reliable. However, considering the expensive installation cost and low utilization rate of the ESS, it may not be the most cost-effective solution. As an alternative, attempts have been made to apply the sharing economy to the energy sector [3,4]. Reference [3] points out the applicability of the sharing economy in 
the energy sector, and offers a proper solution to the problem. It demonstrates that the distributed renewable energies, the ESS, and smart energy management provide an opportunity for increased peer-to-peer interaction. It also shows that sharing the ESS, which might rarely be used to its full capacity by the owner, with neighbors or the community can be beneficial. Therefore, power or energy sharing is an imperative issue for the renewable energy market. As the numbers of prosumers and MGs in modern distribution systems are increasing, power or energy sharing technologies have been discussed in a number of studies from different perspectives. A novel power sharing scheme among multiple microgrids (MMGs) has been proposed for the mitigation of power fluctuation [5]. This aims to improve the cost-efficiency of the ESS when it is used to compensate for the power fluctuation caused by renewables in an MG. In order to improve the system stability, active and reactive power sharing strategies in hierarchical controlled microgrids have been proposed [6].

Herein, the economic operation of MMGs is the main concern. In general, the MG energy management system (MGEMS) is responsible for the economic operation of each MG; however, a higher-level control unit is required to coordinate the power exchange among MMGs. In the two-level energy management system, the goals of MGs and utility are obvious; however, they are rarely mentioned. An MG with a higher time of use (TOU) price and/or with an extreme peak demand needs to coordinate with other MGs. Meanwhile, the utility should reduce the peak demand of the system to mitigate the requirement of infrastructure replacement and investment to meet the highest peak demand. Therefore, an effective power sharing mechanism for MMGs that can consider the benefit for the MG owners, as well as the interest of the utility, is necessary.

Previous studies on energy trading with a cost-effective objective can be summarized as follows. With the objective of maximizing the benefit for MG owners, previous researchers have adopted the bidding or auction mechanism $[7,8]$. In order to balance the selling power and buying power, communication between the MGs and the coordinator is often repeated. Reference [7] develops a community energy market. The MGEMS should determine the role of the MG, i.e., the seller or buyer, in accordance with the power flow direction to the grid after the calculation of the economic dispatch for a single entity. The surplus energy and its price and the insufficient demand can then be identified. The market operator can find the spot price by intersecting the demand and the ascending price rates bid. Reference [8] proposes a naïve auction mechanism. The load agent and generator agent are also determined according to the MG status, e.g., either surplus or deficit generation. Then, the auction agent implements the symmetrical assignment, and if it is not symmetrical, the main grid is required to supplement the difference. References [9-13] regard the global profit as the optimization objective. Reference [9] solves energy trading among interconnected MGs as a Nash bargaining problem. This problem is decomposed into two sequential sub-problems of energy trading and scheduling, and trading payment. The first-step problem is used to minimize the social operation cost and obtain the energy trading amount, while the second-step problem implements the fair allocation of benefits to each participating MG. All of the steps are solved by dual optimization. For distributed optimization, a well-known sub-gradient method is commonly adopted for an iterative solution of the dual problem. However, it is unable to achieve an exact balance between demand and supply with a small number of iterations. Therefore, Ref. [10] proposes a novel linear function submission-based double auction method. This method formulates the individual demand or supply of each prosumer as a linear function by two parameters, where the market-clearing price can be exactly determined by calculating the point at which the two linear functions intersect. However, one parameter of the linear function is the price resilience parameter, which is difficult to estimate. Reference [11] proposes two-level optimal energy management between MMGs with sequentially coordinated operations. Upper level optimization can perform the trading between MGs with surplus energy and the MGs with an energy shortage, to reduce the cost of external trading with the utility. Meanwhile, the lower level implements local optimization of the single MG considering the trading scheduling. Moreover, reference [12] proposes a coordination strategy for optimal power sharing between MMGs in a two-level hierarchical system. Similar to other hierarchical energy management systems, the upper-level center 
implements the coordination strategy to reduce the social cost, while the lower-level MGEMS conducts local optimal energy management. This strategy drastically reduces the calculation burden of the centralized optimization with a large number of MGs. However, it is worth noting that the benefits of the utility are not discussed. Although the stability and economy of the utility are considered, its power exchange structure still requires the actual physical connection, and it is not suitable for a large-area MMG, due to the power loss, as elaborated in [13]. Reference [14] also adopts a two-level energy management system for achieving the goal of the economic operation of MMGs. The difference is that its objective function includes the power loss induced by the power exchange between MGs.

The advantages and disadvantages of the existing methods are summarized in Table 1. Previous studies on power sharing among MMGs have mainly focused on the benefit for MGs only, not for the utility. However, since power sharing between MGs is conducted through distribution lines, this may have an adverse effect on the utility as the number of MGs that participate in the sharing increases. Therefore, this research proposes a power sharing scheme that is beneficial to both MGs and the utility. The two-level hierarchical energy management scheme is adopted, where the power sharing schedule is coordinated by the MG aggregator. Firstly, a mixed integer linear programming (MILP)-based centralized approach for optimal power sharing coordination is proposed. This approach is an extension of the optimal ESS scheduling problem for a single MG, which has previously been proposed by the authors [15]. Although it can provide the optimal power sharing solution, it lacks practical feasibility, due to the large calculation burden as the number of MGs increases. To overcome the significant calculation burden of centralized optimization, a new method for determining the power sharing schedule is proposed. In this approach, the amount of power sharing is assumed to be multiples of the unit amount, and the final power sharing schedule is determined by iteratively finding the best MG pair that exchange a unit amount. The proposed power sharing scheme and the scheduling algorithm are tested with a five MG scenario, and the effectiveness is verified in terms of the operating cost of MGs, the peak demand of utility, and the calculation burden. In addition, the interrelationship between power sharing and the system loss is analyzed when MGs exchange power through the utility network.

Table 1. Advantages and disadvantages of the existing power sharing methods of multiple microgrids (MMGs).

\begin{tabular}{|c|c|c|}
\hline & References $[7,8]$ & References [9-13] \\
\hline Objective & Maximize the benefit for MG owners & Maximize the global profit \\
\hline Advantages & $\begin{array}{c}\text { Guarantee of the maximal benefit for the MG } \\
\text { owners; } \\
\text { less complexity of the clearing or pairing } \\
\text { method in the market. }\end{array}$ & $\begin{array}{l}\text { Ensure the maximal global benefit for all } \\
\text { participants; } \\
\text { realization of better energy utilization in a } \\
\text { local region; } \\
\text { strong controllability for the operator due to } \\
\text { more abundant information collection. }\end{array}$ \\
\hline Disadvantages & $\begin{array}{l}\text { Only feasible for MGs with surplus } \\
\text { renewable energy generation; } \\
\text { require exact estimation of the bidding price. }\end{array}$ & $\begin{array}{l}\text { Heavy computational burden; } \\
\text { high communication cost for information } \\
\text { exchange between the aggregator and MGs. }\end{array}$ \\
\hline
\end{tabular}

\section{Power Sharing Scheme and Scheduling Algorithm}

Figure 1 shows a schematic of the hierarchical energy management system adopted in this paper. In a local MG, an MGEMS measures the power, with smart meters, at the point of common coupling (PCC), as well as the individual devices, such as PV, loads, and the ESS. Based on these measurements and the forecast, MGEMS determines the operation schedule and controls the devices. The MG aggregator coordinates the power exchange between MGs to reduce the total operating cost of the MGs. The MG aggregator communicates with MGEMSs to collect the MG operation data and transmit the optimal power sharing schedule. If MGs are interconnected through a distribution network, power exchange between MGs may affect the network operation. Therefore, the utility or distribution 
system operator (DSO) can review the effect of the power sharing schedules on the distribution network. If congestion is expected in a network, DSO can ask the MG aggregator to adjust the schedule. DSO can also cooperate with the MG aggregators if necessary for better system operation [16].

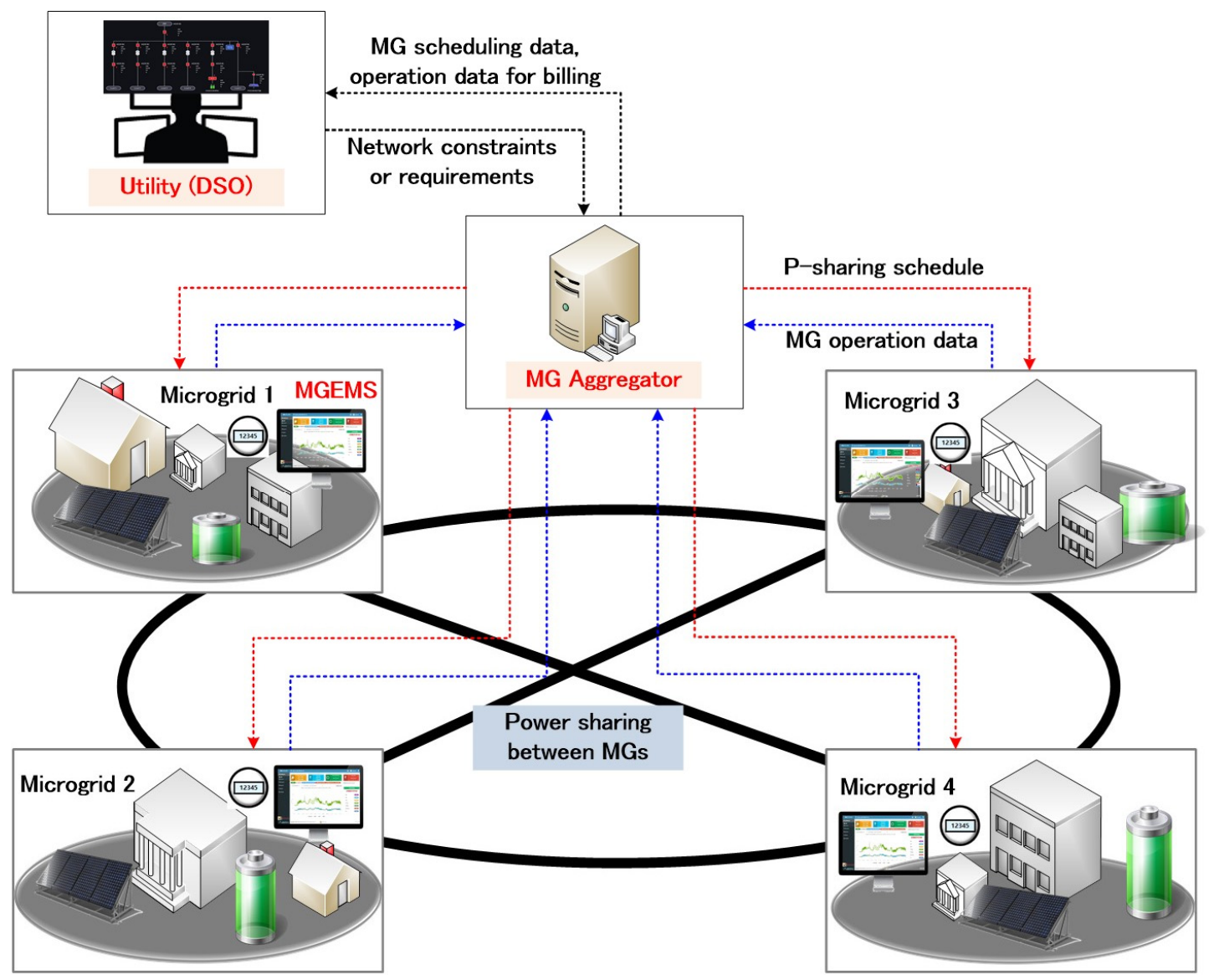

Figure 1. Schematic of the proposed hierarchical energy management system.

\subsection{Local Energy Management by the MGEMS}

Energy management of the individual MG is achieved by optimal ESS scheduling, with the objective of minimizing the operation cost, which includes the energy cost, demand charge, and ESS wear cost. This problem is formulated based on mixed integer linear programming (MILP), as follows:

$$
\begin{aligned}
\min _{M G}= & \sum_{t=1}^{T}\left\{C_{r}(t) \cdot P_{\text {net flow }}(t)+\frac{P_{b}^{+}(\mathrm{t}) / \eta_{d}+P_{b}^{-}(t) \cdot \eta_{c}}{2 \cdot S O C_{\text {max }}-S O C_{\text {min }} \cdot B_{\text {capacity }}} \times \frac{B_{\text {price }}}{B_{\text {cycle }}}\right\} \\
& +I \cdot C_{\text {penalty }} \cdot\left[\max \left\{P_{\text {net flow }}(t)\right\}-P_{\text {demand }}^{\text {contract }}\right]
\end{aligned}
$$

where, $T$ is the specified ESS scheduling horizon, which is selected to be $24 \mathrm{~h}$ herein; $C_{r}(t)$ is the TOU price of time $t ; S O C_{\max }$ and $S O C_{\min }$ represent the maximum and minimum SOC limit, respectively; and $\eta_{d}$ and $\eta_{c}$ are the efficiency of discharge and charge, respectively. Since the electricity bill will be charged based on the measurement at the PCC, the scheduled net flow $P_{\text {net flow }}(t)$ of the PCC considering ESS operation can be defined as:

$$
P_{\text {net flow }}(t)=P_{\text {load }}^{\text {fore }}(t)-P_{p v}^{\text {fore }}(t)-P_{b}^{+}(t)+P_{b}^{-}(t),
$$

where, $P_{\text {load }}^{\text {fore }}(t)$ and $P_{p v}^{\text {fore }}(t)$ are the forecasted load and solar generation at time $t$, respectively, while $P_{b}^{+}(t)$ and $P_{b}^{-}(t)$ represent the discharge and charge power. 
The second term represents the battery wear-cost. The unit wear-cost price of each discharge cycle is obtained by dividing the battery installation $\operatorname{cost}\left(B_{\text {price }}\right)$ by the available cycle number $\left(B_{\text {cycle }}\right)$ for the corresponding depth of discharge (DOD) value. $B_{\text {capacity }}$ is the rated capacity of the ESS. The reason for dividing by 2 in this term is that a cycle of the ESS consists of two process: charging and discharging. The third term of the objective function relates to the demand charge, which is billed at the penalty price $\left(C_{\text {penalty }}\right)$ for the improper usage of power when the maximum net flow during any measured period exceeds the contract power $\left(P_{\text {demand }}^{\text {contract }}\right)$. The integer variable $I$ is introduced to indicate whether the penalty is applied, as given in Equation (3):

$$
I=\left\{\begin{array}{cc}
1 & \max \left\{P_{\text {net flow }}(t)\right\}>P_{\text {demand }}^{\text {contract }} \\
0 & \text { otherwise }
\end{array}\right.
$$

The SOC of the ESS at time $t$ is constrained as follows:

$$
S O C_{\min } \leq S O C(t) \leq S O C_{\max }
$$

where, the SOC of the ESS at any time $t$ can be calculated by the following equation:

$$
\operatorname{SOC}(t+\Delta t)=\operatorname{SOC}(t)-\frac{\left(\frac{P_{b}^{+}(t)}{\eta_{d}}-P_{b}^{-}(t) \cdot \eta_{c}\right) \cdot \Delta t}{B_{\text {capactiy }}} \times 100 .
$$

Moreover, the instantaneous discharge/charge power of the ESS depends on the capacity of the power conditioning system (PCS). The constraint for this physical limit is given as follows:

$$
\begin{gathered}
0 \leq P_{b}^{+}(t) \leq \gamma(t) \cdot P_{b}^{\max } \\
0 \leq P_{b}^{-}(t) \leq(1-\gamma(t)) \cdot P_{b}^{\max },
\end{gathered}
$$

where, $P_{b}^{\max }$ is the rated power capacity of the PCS and $\gamma(\mathrm{t})$ is a binary variable that is introduced to prevent the charge and discharge from occurring simultaneously:

$$
\gamma(t)=\left\{\begin{array}{cc}
1 & \text { if discharge } \\
0 & \text { if charge }
\end{array}\right.
$$

In order to calculate the demand charge exactly, the power flow at the PCC is measured periodically. In addition, the maximum net flow at the PCC should be formulated into the problem and recorded. A detailed process has been presented by the authors in the previous study [15].

\subsection{Power Sharing Scheduling among MGs Coordinated by the MG Aggregator}

\subsubsection{Power Sharing Scheme Adopted in this Research}

Power exchange among MMGs can be achieved in a variety of ways. For the MGs with large-capacity renewable energy sources (RESs), the surplus energy can be sent to the other MGs through the direct physical connection line, or indirectly through the distribution lines. For the MGs without adequate RESs but with controllable distributed generations (DGs), the low-cost generation units will provide the power to the other MGs for the appropriate revenue. This research considered MGs with small-capacity RESs and ESS, but no other controllable resources. For this type of MG, the ESS is actively advocated for power sharing, instead of the way in which the MG buys electricity from the grid, and sells to other MGs directly. It can help the MG owners to achieve benefits in power trading, similar to other power trading structures; furthermore, it can contribute to reducing the peak demand for the utility. For this, the MG that sends power should reduce its net flow by as much power 
as it sends to other MGs by generating additional power, reducing the demand, or discharging the power from ESS.

In this study, it is assumed that the electricity charges for individual MGs are calculated by taking into account power sharing and the physical power consumption. Therefore, the power exchange between MGs, as well as the net flow of MGs at the PCC, are recorded by the MG aggregator and reported to the utility. Figure 2 shows the schematics of two power sharing schemes. It is assumed that the expected net flows of the two MGs are the same at $100 \mathrm{~kW}$, as shown in Figure 2a. If the total cost of two MGs can be reduced by transferring $20 \mathrm{~kW}$ of power from MG A to MG B, there are two different ways to conduct the sharing. Figure $2 b$ shows one way, in which MG A transfers $20 \mathrm{~kW}$ to MG B without controlling any resources. In this case, the electricity charge will be calculated as MG A used $120 \mathrm{~kW}$ and MG B used $80 \mathrm{~kW}$. However, the actual power flows from the utility to the two MGs are the same as those before the power sharing. Therefore, in this scheme, there is no benefit for the utility, even if the operating cost of MGs can be reduced. Figure 2c shows the other way, in which MG A controls its resources to decrease its net flow by $20 \mathrm{~kW}$; the same amount as it sends to MG B. In this scheme, the power sharing between MGs reduces the power from the utility to $180 \mathrm{~kW}$, which is beneficial to the utility, especially in peak hours. In this study, the latter is adopted for the benefit of both MGs and the utility. Since it is assumed in this research that ESS is the only controllable resource in the MG, the sending MG should discharge power from the ESS by at least the same amount of power as it sends to other MGs.

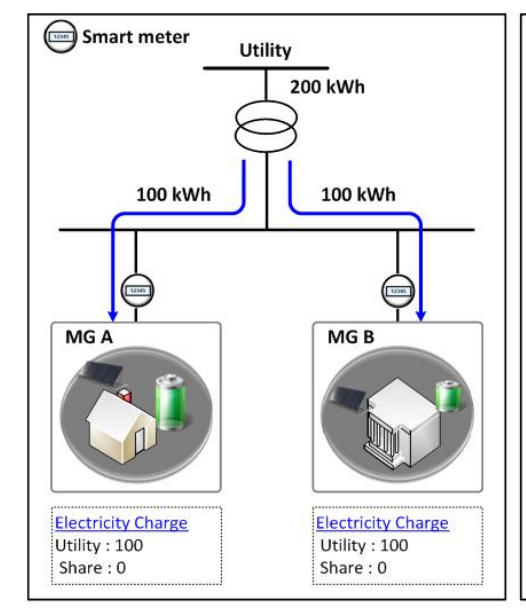

(a)

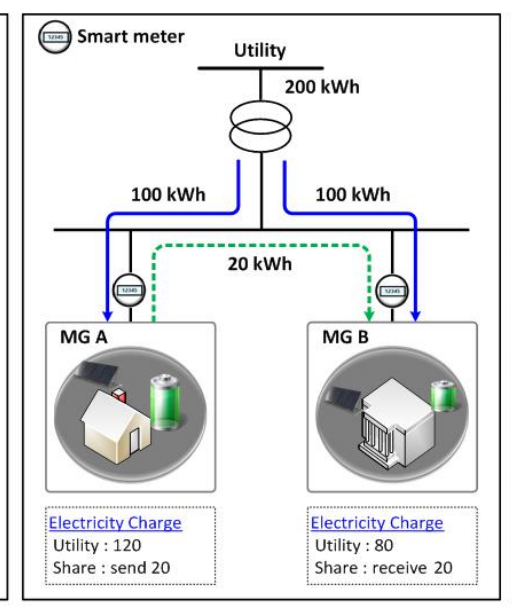

(b)

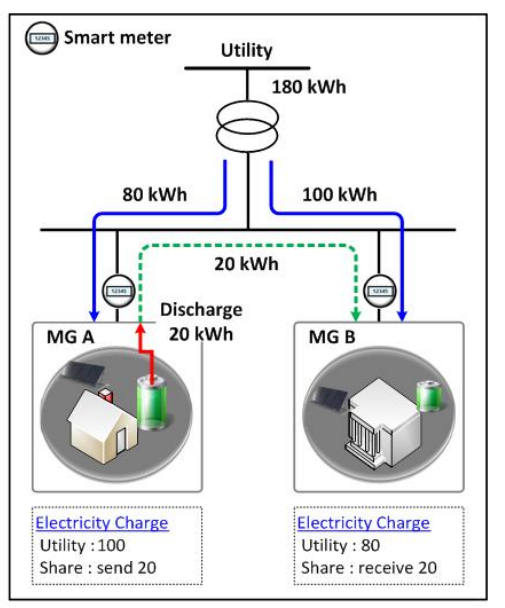

(c)

Figure 2. Power flow and billing information of the two power sharing schemes: (a) without power sharing; (b) power sharing without physical control of resources; (c) power sharing with physical control of resources.

\subsubsection{MILP-Based Centralized Power Sharing Scheduling Method}

The objective of power sharing is to minimize the total operation cost $\left(C_{T}\right)$ of multiple MGs, which can be represented as follows:

$$
\min C_{T}=\sum_{i=1}^{N_{M G}} C_{M G, i}
$$

The problem can be formulated by MILP similarly to the single MG scheduling problem, with minor modifications. The electricity consumption measured at the utility's meter is the same as in Equation (2). However, the actual operation cost of the individual MG should be determined considering not only the data recorded by the meter, but also the power sharing with other MGs. The aggregator has the responsibility of reporting the information of power sharing among MGs, 
or between the external grid and the utility. The final electricity billing can be calculated in the same way as in Equation (1), but the net flow of each MG should be reformulated considering the power sharing schedule, as follows:

$$
P_{\text {net flow }}^{\text {billing }}(i, t)=P_{\text {load }}^{\text {fore }}(i, t)-P_{p v}^{\text {fore }}(i, t)-P_{b}^{+}(i, t)+P_{b}^{-}(i, t)-P_{m}^{-}(i, t)+P_{m}^{+}(i, t),
$$

where, $P_{m}^{-}(i, t)$ and $P_{m}^{+}(i, t)$ are referred to as the receiving power and sending power of MG $i$ at time $t$, respectively. The constraints for each MG, presented by Equations (3)-(8), are applied without modification. In order to support the proposed power sharing scheme, a new constraint for the ESS discharge power is added as follows:

$$
0 \leq P_{m}^{+}(i, t) \leq P_{b}^{+}(i, t)
$$

In addition, for each MG, the behavior of the sending and receiving power cannot occur simultaneously. To achieve it, the constraints are added as follows:

$$
\begin{gathered}
0 \leq P_{m}^{+}(i, t) \leq k(i, t) * P_{\text {inf }} \\
0 \leq P_{m}^{-}(i, t) \leq(1-k(i, t)) * P_{\text {inf }},
\end{gathered}
$$

where, $P_{\text {inf }}$ is a positive constant that is large enough not to make practical restrictions on power sharing, and $k(i, t)$ is a binary variable that is defined as follows:

$$
k(i, t)=\left\{\begin{array}{cc}
1 & \text { if } \mathrm{MG} i \text { is the sending power at time } t \\
0 & \text { if } \mathrm{MG} i \text { is the receiving power at time } t
\end{array} .\right.
$$

Moreover, the sending and receiving power must be balanced at all times, and this can be presented as follows:

$$
\sum_{i=1}^{N_{M G}} P_{m}^{+}(i, t)=\sum_{i=1}^{N_{M G}} P_{m}^{-}(i, t) .
$$

\subsubsection{New Power Sharing Scheduling by Iterative Optimal Pairing}

Although centralized optimization can provide the optimal power sharing solution, it lacks practical feasibility, due to the large calculation burden caused by the increased difficulty of time-dependence between variables. As the number of MGs increases, the optimal solution may not be found within a limited time. Although distributed optimization may be effective in solving such a problem, it is difficult to fully consider the time-dependent variables with multiple constraints.

To overcome the significant calculation burden of centralized optimization and the increased difficulty of the time-dependent variable with multiple constraints, a new method for determining the power sharing schedule is proposed. In practice, the amount of power sharing schedule does not need to be precise to the decimal. Therefore, this paper assumes that MGs share power in multiples of a unit amount. Based on this, the optimal power sharing schedule can be determined by repeatedly finding the best two MG pairs that exchange the unit amount of power. The concept and detailed implementation process are as follows.

Firstly, for the MGs that have agreed to participate in the power sharing, the optimal ESS operation schedule is calculated without considering the power sharing. The operation cost of each MG with the self-ESS-scheduling is obtained, and is regarded as the base operation cost of each MG. Then, for each MG, the ESS schedule and associated operating cost are recalculated for the cases in which it sends and receives the unit amount power, respectively. It is not hard to imagine that the operating cost of the MG that sends power will be increased, due to the increased electricity billing and additional cost of efficiency loss and wear cost of the ESS, while the operation cost of the MG that receives power will be 
decreased. If an MG is assumed to receive a power of unit amount, the net demand is adjusted as shown in Equation (16) before the calculation of the new ESS schedule. Equation (17) ensures that if the receiving power exceeds the net demand, the power not consumed by the load is charged into the ESS:

$$
\begin{gathered}
P_{\text {netdemand }}^{\text {new }}(i, t)=P_{\text {netdemand }}^{\text {old }}(i, t)-P_{\text {sharing }}^{\text {unit }} \\
P_{b}^{-}(i, t)=P_{\text {netdemand }}^{\text {new }}(i, t) \quad \text { if } P_{\text {netdemand }}^{\text {new }}(i, t)<0,
\end{gathered}
$$

where, $P_{\text {shiring }}^{\text {unit }}$ is the predetermined unit amount of power sharing; $P_{\text {netdemand }}(i, t)$ is the net demand of MG $i$ at time $t$ considering the forecasted load, forecasted PV generation, and power sharing schedule determined up to the calculation of the last iteration; and $P_{\text {netdemand }}^{\text {new }}(i, t)$ is the net demand considering the assumed receiving power schedule in this calculation iteration.

If an MG is assumed to send power at some time, the net demand will be increased by the unit amount, as presented in Equation (18). Since the sending power should be from the discharge of the ESS, the discharge schedule at the time should be greater than or equal to the accumulated sending power, $P_{m, a s s u m e d}^{+}(i, t)$, as shown in Equation (19):

$$
\begin{gathered}
P_{\text {netdemand }}^{\text {new }}(i, t)=P_{\text {netdemand }}^{\text {old }}(i, t)+P_{\text {sharing }}^{\text {unit }} \\
P_{b}^{+}(i, t) \geq P_{m \text {,assumed }}^{+}(i, t) .
\end{gathered}
$$

After calculating the new operation cost of all MGs for all possible scenarios, the deviation from the base operation cost will be calculated. Then, one-to-one symmetrical pairing between the sending-power MG and the receiving-power MG is implemented. If the number of MGs is $N$ and the scheduling time horizon is $T$, there exist $N \times(N-\mathbf{1}) \times \mathbf{T}$ power sharing pairs. If the cost reduction of a receiving-power $\mathrm{MG}$ is greater than the cost increase of the sending-power $\mathrm{MG}$, the total operation cost of MGs can be decreased. In each calculation iteration, the sharing pair that reduces the cost the most will be selected. Since the amounts of sending and receiving power are exactly the same as the unit amount, balance between the two is always ensured. Figure 3 illustrates the process employed to select the best power sharing pair in each iteration.

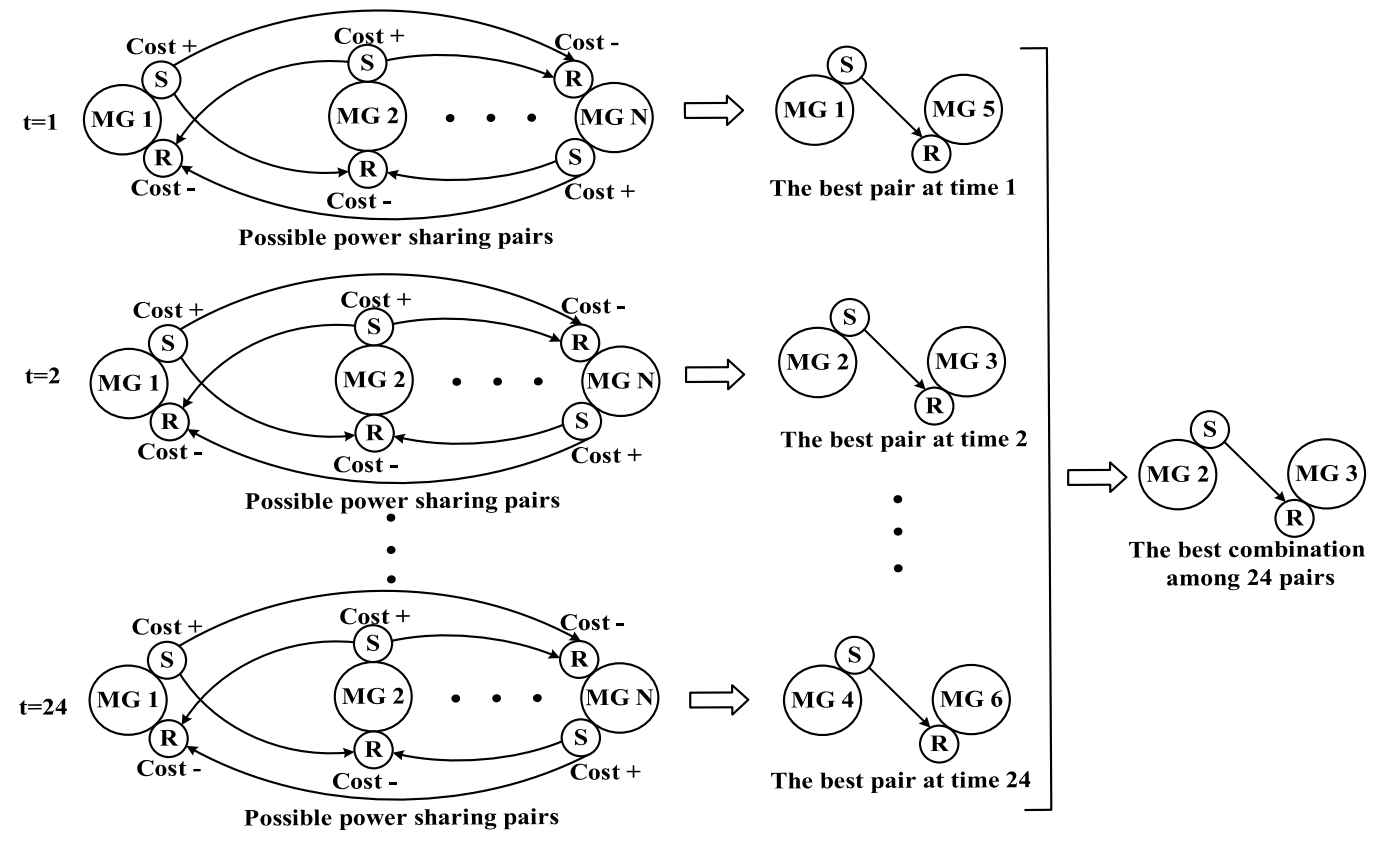

Figure 3. The process employed to select the best power sharing pair in each iteration. 
The net demand of the selected MGs and time are updated as follows:

$$
\begin{gathered}
P_{\text {netdemand }}\left(i_{\text {send }}, t_{\text {share }}\right)=P_{\text {netdemand }}\left(i_{\text {send }}, t_{\text {share }}\right)+P_{\text {sharing }}^{\text {unit }} \\
P_{\text {netdemand }}\left(i_{\text {receive }}, t_{\text {share }}\right)=P_{\text {netdemand }}\left(i_{\text {receive, }}, t_{\text {share }}\right)-P_{\text {sharing }}^{\text {unit }}
\end{gathered}
$$

where, $\boldsymbol{i}_{\text {send }}$ and $\boldsymbol{i}_{\text {receive }}$ are the selected sending and receiving MG indices, respectively, and $\boldsymbol{t}_{\text {share }}$ is the time index of the selected sharing pair. In the next iteration, the ESS schedule and operating cost of the two MGs selected are recalculated with the updated net demand, and the best power sharing pair is then selected. This assumption-selection-update process is repeated until any sharing pair can no longer reduce the cost, and the whole process is summarized in the Algorithm 1.

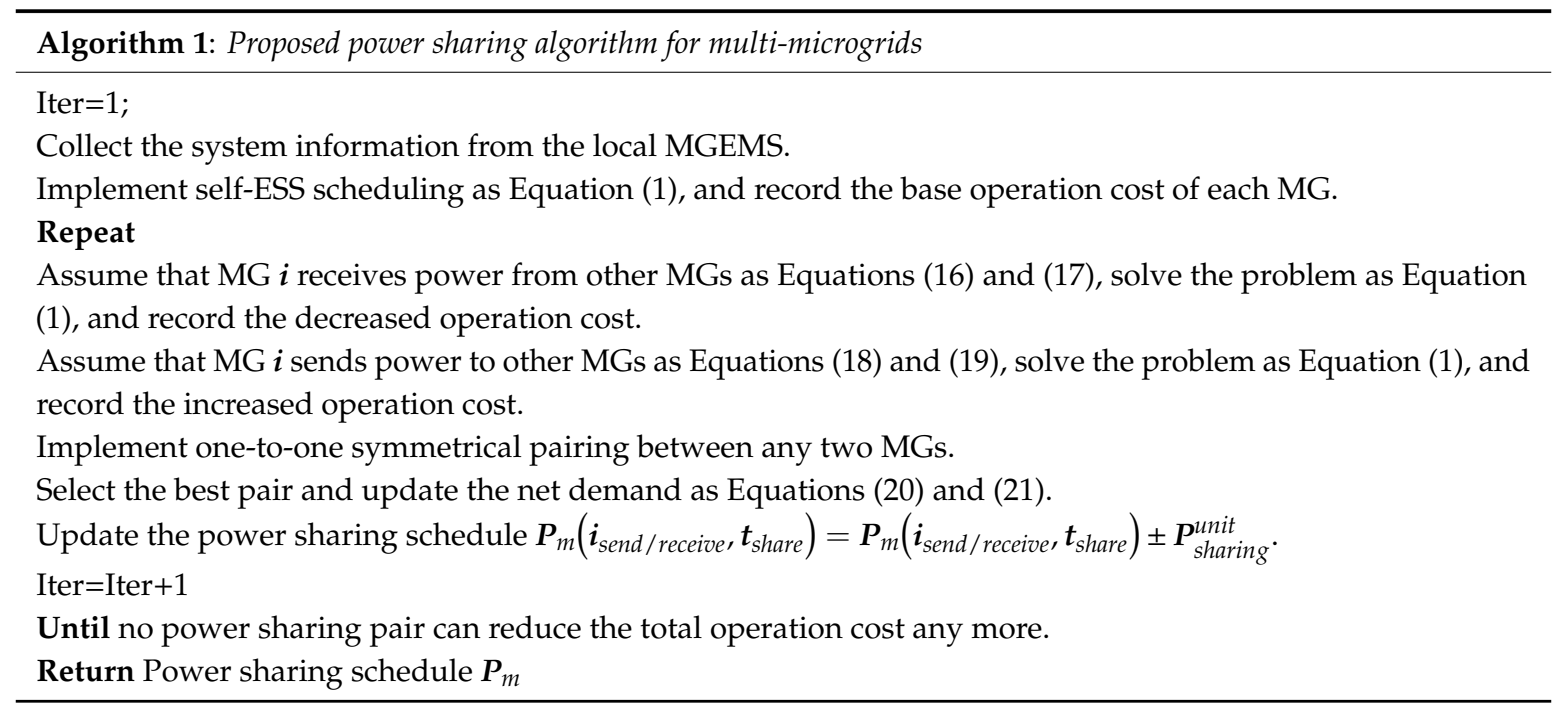

If the MG aggregator has detailed operational information for all MGs participating in power sharing, the above-mentioned process may be performed by the MG aggregator. The proposed method can also be implemented in a distributed manner, since individual MGs do not require any information from other MGs to recalculate their operating costs assuming power sharing. In this distributed approach, the MG aggregator only collects increases and decreases in operating costs from individual MGs. When the optimal sharing pair is selected by the MG aggregator, only the selected MGs recalculate the operating costs using the updated net demand. Since only the operating costs of the two selected MGs need to be recalculated at each iteration, the computation time will not increase drastically, even if the number of MGs participating in power sharing increases.

\subsection{TOU Price Conditions for Power Sharing for Energy Cost Saving Purposes}

There are two main cases where power sharing between MGs can occur. The first case is when the load of a certain MG is expected to exceed the contract demand. Since the penalty cost is generally much larger than the TOU price, it is clear that, in this case, the power sharing between MGs can reduce the overall operating cost. The second case is when the TOU prices of MGs are different, so that the energy cost can be reduced through power sharing. However, power sharing for this purpose cannot easily occur, unless the TOU difference between the sending and receiving MGs is large enough. Considering the losses during the charge/discharge process, the MG that sends power to another MG should charge its ESS at another time, and the required charging energy is as follows:

$$
P_{b}^{-}\left(i_{\text {send }}, t_{\text {char }}\right)=\frac{1}{\eta_{d} \cdot \eta_{c}} P_{m}^{+}\left(i_{\text {send }}, t_{\text {share }}\right)
$$


In addition, the wear cost for using ESS should also be considered. From the definition in

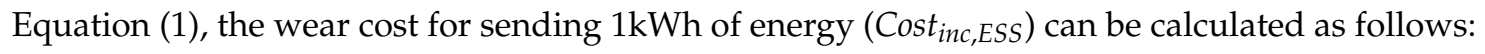

$$
\operatorname{Cost}_{\text {inc }, \text { ESS }}=\frac{1 / \eta_{d}}{B_{\text {capacity }} \cdot\left(S O C_{\max }-S O C_{\min }\right)} \cdot \frac{B_{\text {price }}}{B_{\text {cycle }}} .
$$

Therefore, power sharing for energy cost saving purposes can occur when the TOU price of the receiving MG $\left(T O U_{R}\right)$ is higher than that of the sending MG $\left(T O U_{S}\right)$ by enough to compensate for the charging cost and the battery wear cost, as follows:

$$
\operatorname{TOU}_{R}\left(t_{\text {share }}\right)>\operatorname{TOU}_{S}\left(t_{\text {char }}\right) \cdot \frac{1}{\eta_{d} \cdot \eta_{c}}+\operatorname{Cost}_{\text {inc,ESS }} .
$$

\section{Simulation Results and Discussions}

\subsection{Simulation Setup and Scenario}

To demonstrate the effectiveness of power sharing between MGs, and to verify the performance of the proposed power sharing scheduling method, the 5-MG system is considered. The 5-MG system adopts the same power sharing structure described in Figure 1, where the net flow of the MGs is measured by smart meters. It is assumed that each microgrid has one ESS and a PV generator. For simplicity of analysis, the size of the PV generator is assumed to be small enough not to result in a reverse power flow. In other words, the power from the PV generator is assumed to be consumed by the local load in each MG. Figure 4 shows the net demand of five MGs, whose values are based on the actual measurements of campus buildings, with slight modification for the simulation purpose. Table 2 summarizes the contract demand value and the parameters of the ESS.



Figure 4. Net demands of the microgrids (MGs).

Figure 5 shows the TOU price of the MGs in Korean Won (KRW) per kWh, which is based on the electricity rate applied to educational customers in Korea [17]. We deliberately adjusted the TOU rate of MG 5 at 3:00 so that it was higher than the actual value, so that power sharing for energy cost savings could occur. If the TOU of MG 5 is high enough, MGs 1 or 4 that have a large ESS may send power to MG 5 to reduce the overall energy cost. Assuming that MG 1 or 4 charges its ESS at off-peak hours, the minimum value of the TOU of MG 5 at 3:00 required for this power sharing to occur can be calculated as shown in Equation (25). Therefore, in this simulation, the TOU of MG 5 at 3:00 was set to 80.

$$
\operatorname{TOU}_{M G 5}\left(t_{\text {sharing }}=3\right)>49.8 \times \frac{1}{0.95 \times 0.95}+\frac{1 / 0.95}{500 \times(0.9-0.1)} \times \frac{30,000,000}{3250}=79.5
$$


Table 2. Simulation conditions.

\begin{tabular}{cccccc}
\hline Parameter & MG 1 & MG 2 & MG 3 & MG 4 & MG 5 \\
\hline$P_{\text {demand }}^{\text {contract }}(\mathrm{kW})$ & 400 & 200 & 200 & 500 & 400 \\
$B_{\text {capacity }}(\mathrm{kWh})$ & 500 & 50 & 50 & 500 & 250 \\
$P_{b}^{\text {max }}(\mathrm{kW})$ & 500 & 50 & 50 & 500 & 250 \\
$B_{\text {price }}(\mathrm{KRW})$ & $30,000,000$ & $5,000,000$ & $5,000,000$ & $30,000,000$ & $20,000,000$ \\
$B_{\text {cycle }}$ & 3250 & 3250 & 3250 & 3250 & 3250 \\
$C_{\text {penalty }}(\mathrm{KRW})$ & 6090 & 6090 & 6090 & 6090 & 6090 \\
SOC $_{\text {max }}(\%)$ & 90 & 80 & 80 & 90 & 90 \\
SOC $_{\text {min }}(\%)$ & 10 & 20 & 20 & 10 & 10 \\
SOC $_{\text {init }}(\%)$ & 50 & 50 & 50 & 50 & 50 \\
SOC $_{\text {target }}(\%)$ & 50 & 50 & 50 & 50 & 50 \\
$\eta_{d}$ (p.u.) & 0.95 & 0.95 & 0.95 & 0.95 & 0.95 \\
$\eta_{c}$ (p.u.) & 0.95 & 0.95 & 0.95 & 0.95 & 0.95 \\
\hline
\end{tabular}

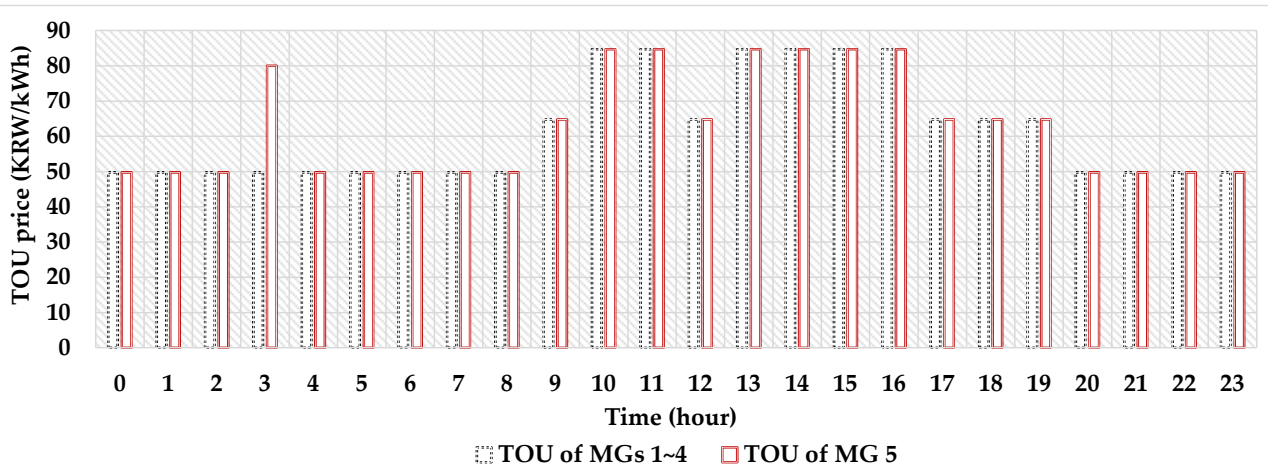

Figure 5. The time of use (TOU) prices of the MGs.

\subsection{MG Operation without Power Sharing}

First, the operational results according to the optimal schedule of the individual MG will be described, without considering power sharing among MGs. In other words, the ESS in each MG is scheduled by only considering its own operating cost. Figure 6 shows the optimal ESS schedule for the five MGs, while Figure 7 shows the net flow of each MG considering the ESS operation. It is observed that MGs 1, 4, and 5, which have a sufficient battery capacity, can reduce the operating costs by discharging during high TOU and high load periods, and charging during low TOU periods. However, for MGs 2 and 3, the net flow exceeds the contract demand, even though the ESS fully discharges at the peak load, due to the capacity limitation. Therefore, without power sharing, MGs 2 and 3 would be subject to large penalties.

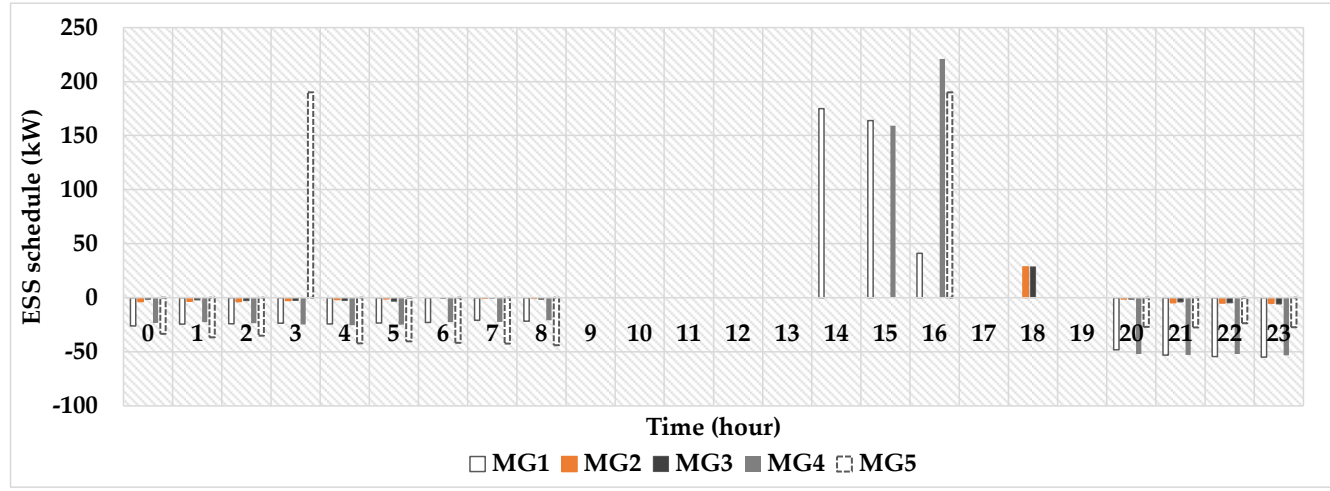

Figure 6. Optimal energy storage system (ESS) scheduling of each MG under a single operation. 


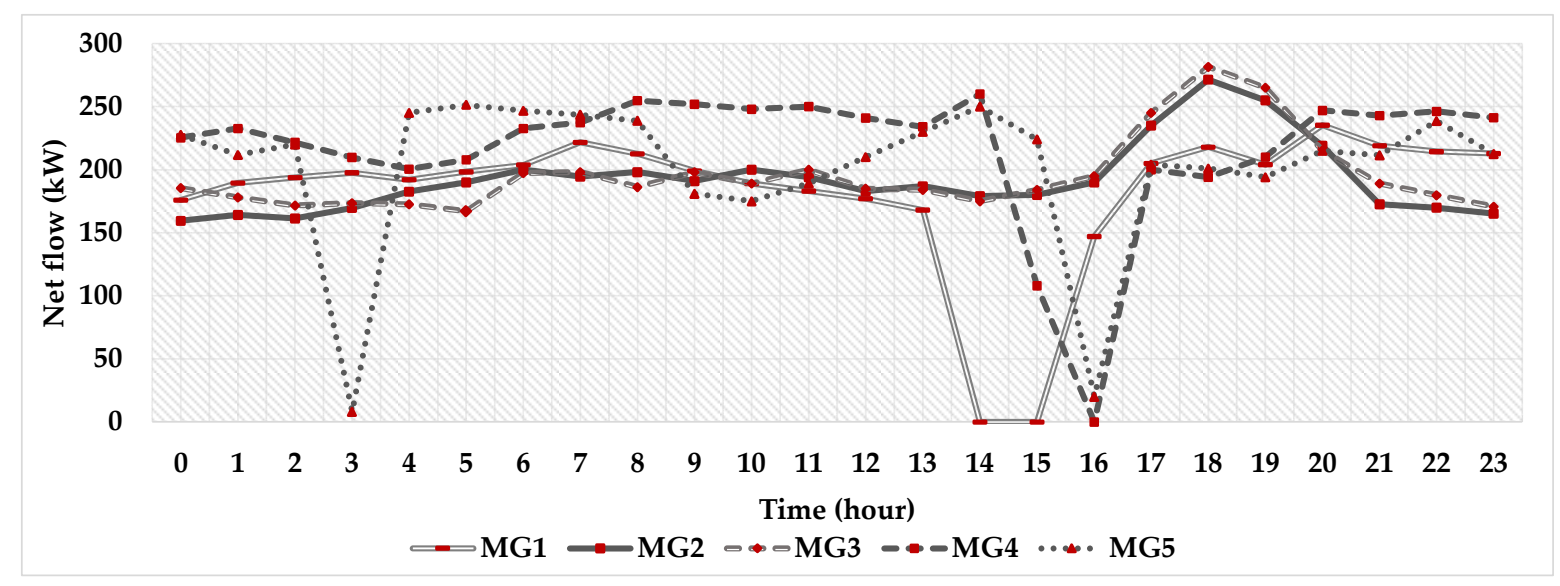

Figure 7. Net flows of the five MGs with the self-ESS-scheduling.

\subsection{MMG Operation Considering Power Sharing among MGs}

This section describes the ESS scheduling considering the power sharing between MGs, and shows the corresponding operation results. Figure 8 shows the power sharing schedule for five MGs determined by the proposed method, where the unit amount $\left(P_{\text {sharing }}^{\text {unit }}\right)$ was chosen to be $20 \mathrm{kWh}$. The positive value in the figure indicates the sending power, while the negative value represents the receiving power. As expected, MGs 2 and 3 will receive power from other MGs during hours when the net flow exceeds the contract demand (between 17:00 and 20:00). MGs 2 and 3 can avoid penalties with the help of other MGs, resulting in a reduction in the overall operating cost of the MGs. In addition, we can observe the schedule in which MG 5 receives power from MGs 1 and 4 at 3:00. As described above, the TOU of MG 5 at 3:00 was set very high, compared to the other MGs. Therefore, this power sharing is more advantageous in terms of the overall operating cost than MG 5 purchasing power from the utility or using its own ESS, even considering the charge/discharge loss, and the wear cost of the ESSs of MGs 1 and 4.

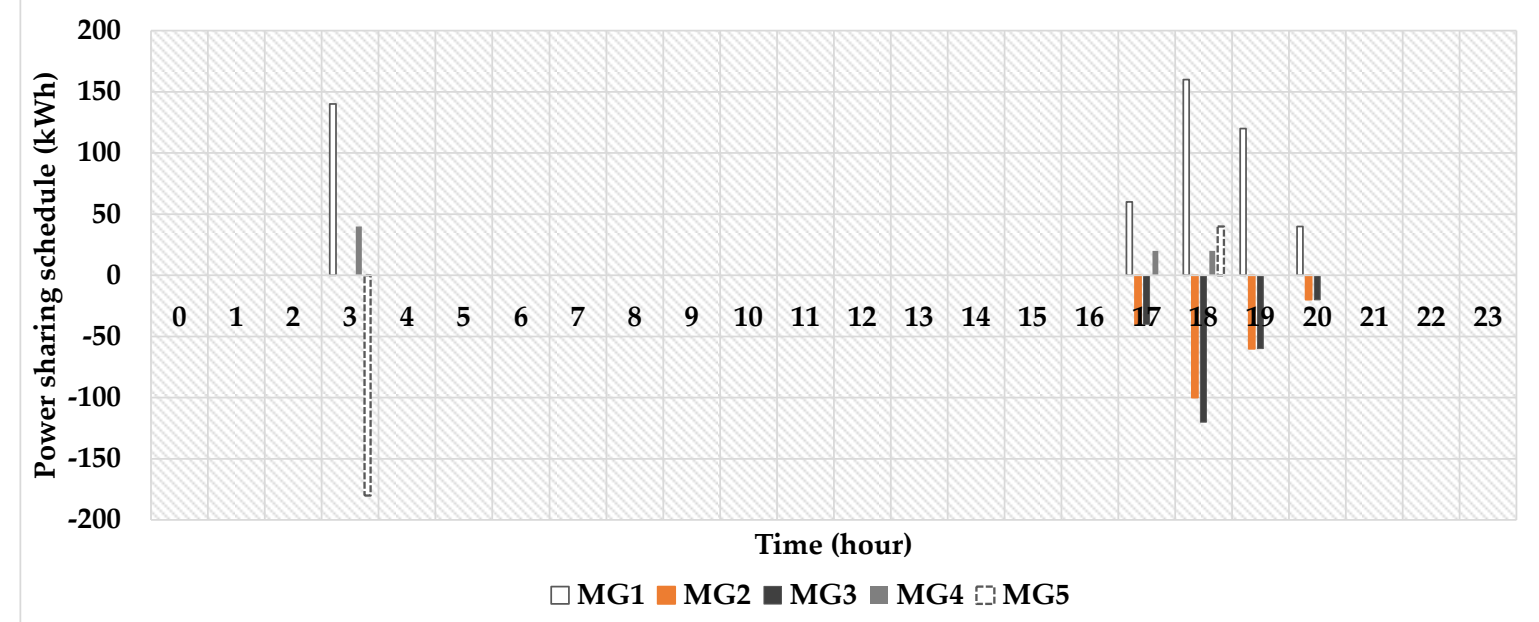

Figure 8. Power sharing schedule determined by the proposed method.

Once the power sharing schedule is determined, the EMS of each MG recalculates the schedule of the ESS accordingly. Figure 9 shows the ESS operation schedule of the MGs considering the power sharing. In the case of MG 1, which sends the most power to other MGs, the discharge schedule is shifted from its highest TOU period of 13:00-16:00 to 17:00-20:00, when MGs 2 and 3 require power. The ESSs in MGs 2 and 3 do not discharge, even at their peak load, since they can receive power from 
other MGs. It can also be seen that, at 3:00, the ESSs in MGs 1 and 4 discharge to send the power to MG 5 , so that the discharge of the ESS in MG 5 decreases accordingly.

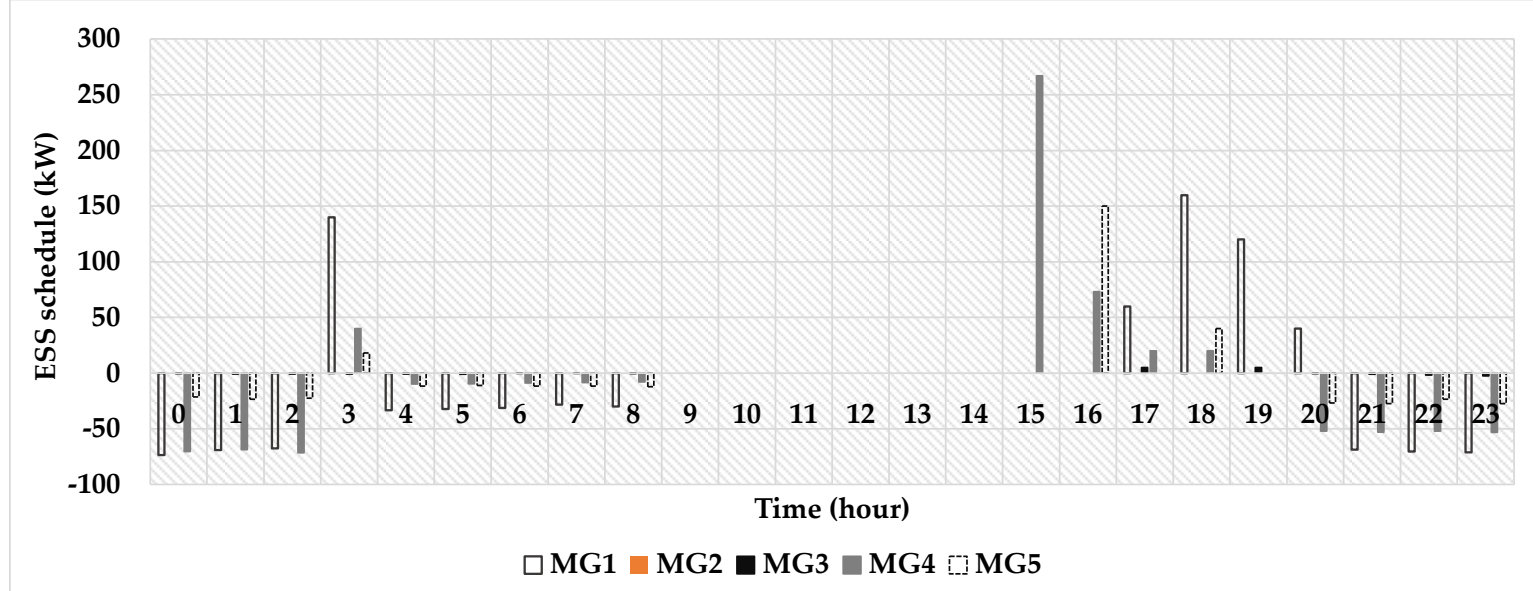

Figure 9. ESS scheduling considering the power sharing scheduling.

Figure 10 shows the equivalent net flow of each MG defined by Equation (10), which is used in the electricity bill calculation considering power sharing. It can be seen that the net flows of MGs 2 and 3 are kept below the contract demand at all times. Table 3 compares the operating cost of MGs with and without considering power sharing. The operating costs of MGs 2 and 3 were drastically decreased after power sharing, because, with the help of other MGs, the penalty could be avoided. In the case of MG 5, which received power from other MGs at its high TOU time, the operating cost was slightly reduced. In contrast, the operating costs of MGs 1 and 4 increased, because their ESSs were used to send the power to other MGs, instead of reducing their own demand during the highest TOU period. However, it is important to note that through power sharing, the total operating costs were reduced by about $38.7 \%$. These profits of power sharing should be distributed to MGs according to their contributions. In other words, the receiving MGs should compensate the sending MGs for their sharing. There are many ways of distributing profits, but this matter is beyond the scope of this paper, and will not be discussed here.

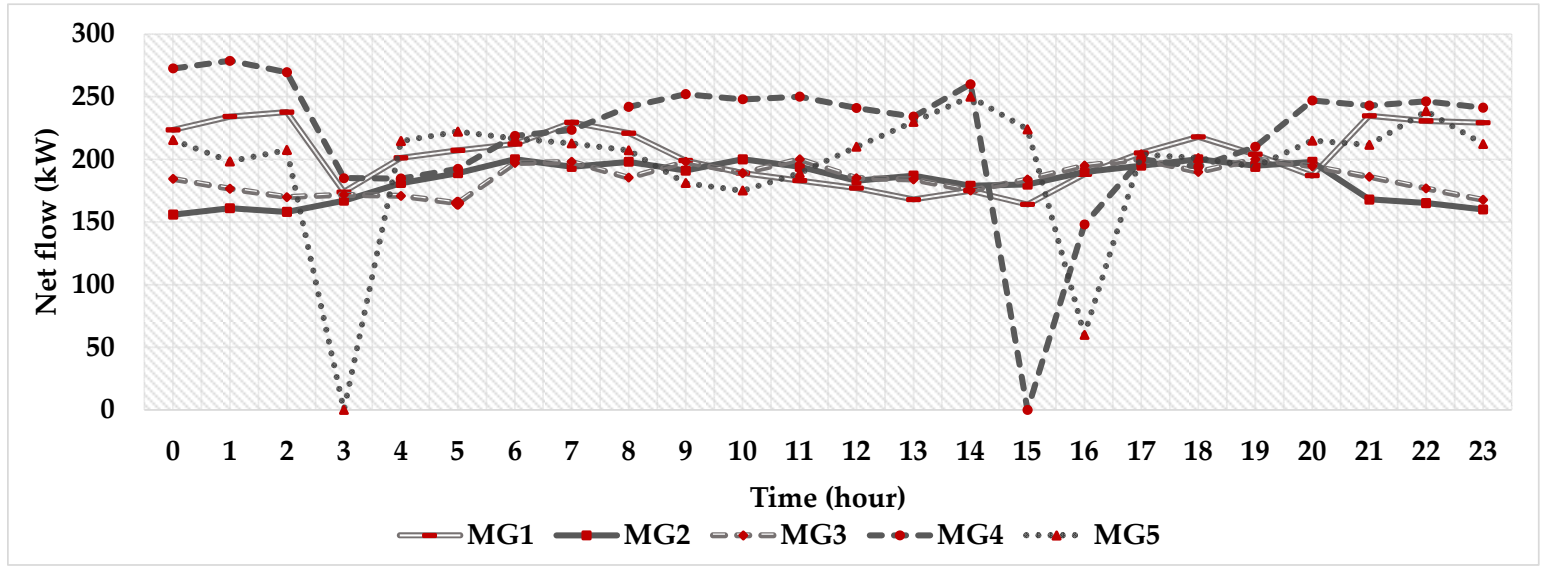

Figure 10. Equivalent net flow of the MGs for the electricity bill calculation considering power sharing. 
Table 3. Operating costs of the MGs with and without considering power sharing.

\begin{tabular}{ccc}
\hline \multirow{2}{*}{ MG Index } & \multicolumn{2}{c}{ Operating Cost (KRW) } \\
\cline { 2 - 3 } & Without Power Sharing & With Power Sharing \\
\hline MG 1 & $261,489.5$ & $303,422.3$ \\
MG 2 & $723,415.0$ & $272,469.2$ \\
MG 3 & $788,480.5$ & $275,884.3$ \\
MG 4 & $319,145.2$ & $325,318.0$ \\
MG 5 & $301,811.8$ & $291,757.4$ \\
Total & $2,394,342.0$ & $1,468,851.2$ \\
\hline
\end{tabular}

For the utility that supplies electricity to the MGs, power sharing may seem to have no direct benefit; rather, power sharing between MGs may reduce the sales revenue of the utility. However, power sharing can help the utility in other ways, by reducing peak loads and flattening load profiles. This allows utilities to defer the construction of transmission and distribution facilities, and avoid the use of expensive generators.

Figure 11 shows the total power supplied by the utility to the five MGs. First, the peak net demand of the day was $1223 \mathrm{~kW}$ at 18:00, assuming that the ESSs were not operating. Next, when the ESSs were operated without considering power sharing, the net demands of 14:00-16:00 were greatly reduced, but the peak demand supplied by the utility was still high, being $1166 \mathrm{~kW}$ at $18: 00$. This is because, as explained earlier, the ESS in one MG is not operated, to help reduce the demand of the other MGs. Finally, when power sharing between MGs was conducted, the net demand at 18:00 was lowered to about $1003 \mathrm{~kW}$. However, in order to discharge the ESS at the peak load hours of MGs 2 and 3, the charges of the ESSs of MGs 1, 4, and 5 at different times were increased, so the maximum demand of the day was about $1058 \mathrm{~kW}$ at 7:00. In summary, power sharing between MGs could reduce the peak demand from the utility perspective by about $9.3 \%$. Although the quantitative effect of peak demand reduction may vary with various conditions, such as load patterns, TOU, and ESS capacities, the simulation results show that power sharing benefits utilities, as well as the MGs.

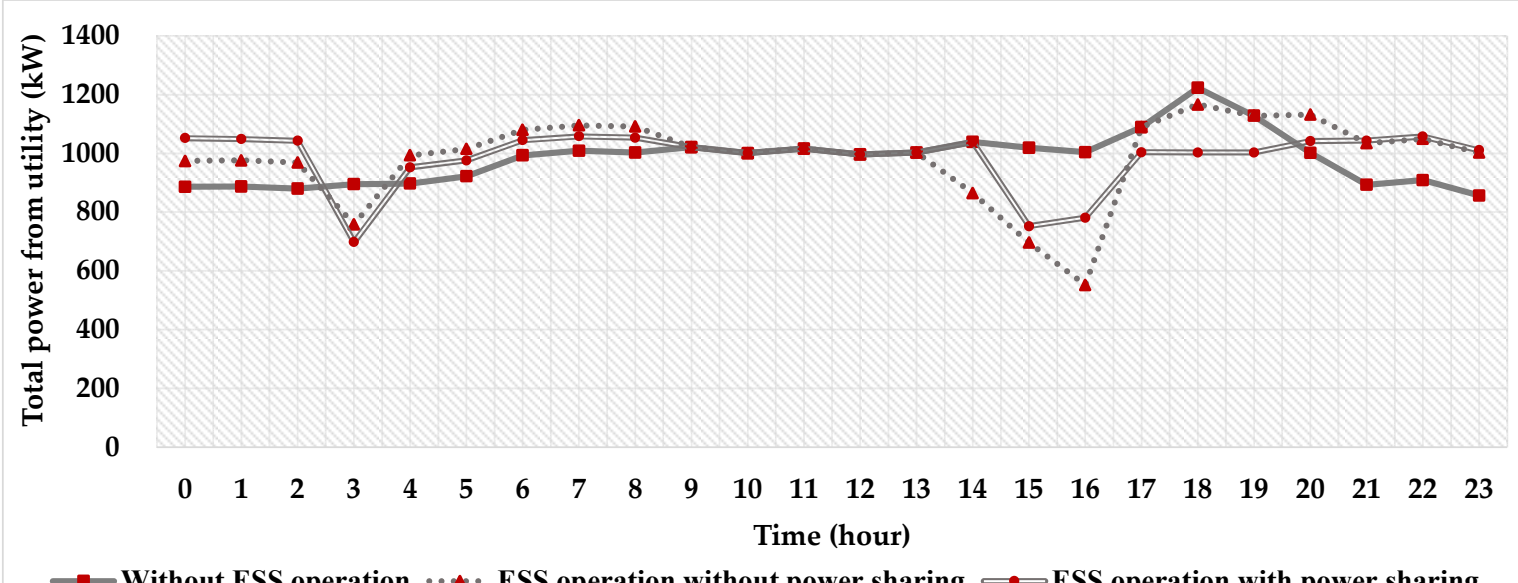

Figure 11. Total electricity supplied by the utility according to the different MG operation schemes.

\subsection{Comparison of Centralized Optimization and the Proposed Power Sharing Scheduling Method}

Table 4 compares the results of the two power sharing scheduling methods-centralized optimization and the proposed method-in terms of the total operating cost and computation time. With the proposed method, the overall operating cost increased slightly, compared to the centralized optimization-based solution. This is because the power sharing schedule of the proposed method is based on multiples of the unit amount. However, the difference in operating costs between 
the two methods was only about $1 \%$. This cost difference is acceptable enough, considering the large reduction in the computation time. In this 5-MG scenario, the computation time of centralized optimization was nearly $2 \mathrm{~h}$, while with the proposed method, it was only about $2.4 \mathrm{~min}$.

Table 4. Performance comparison of the two power sharing methods.

\begin{tabular}{ccc}
\hline Parameter & Centralized Optimization & Proposed Method \\
\hline Total operating cost (KRW) & $1,453,907.2$ & $1,468,851.2$ \\
Computation time (s) & 7180.3 & 205.6 \\
\hline
\end{tabular}

In order to further verify the effectiveness of the proposed method in reducing the calculation burden, simulations were conducted based on various scenarios with different numbers of MGs from two to ten. The computation time depends not only on the number of MGs, but also on the complexity of the input data, such as the load pattern and TOU of the MGs. Therefore, we carefully selected the input data to avoid a loss of generality, and Figure 12 shows the representative simulation results. Considering the practical applicability, we set the simulation to terminate when the computation time exceeded three hours. When the number of MGs was two or three, both methods found a solution within 2.5 minutes. However, when the centralized optimization method was used and the number of MGs was more than four, the computation time increased rapidly. Furthermore, for the cases of more than seven MGs, this method did not even solve the problem within three hours. In contrast, when the proposed method was used, the computation time did not increase significantly, even if the number of MGs was increased. For the 10-MG simulation case, the problem could be solved in about 5 min with the proposed method. Therefore, in terms of the practical applicability, the proposed method is much more useful than centralized optimization.

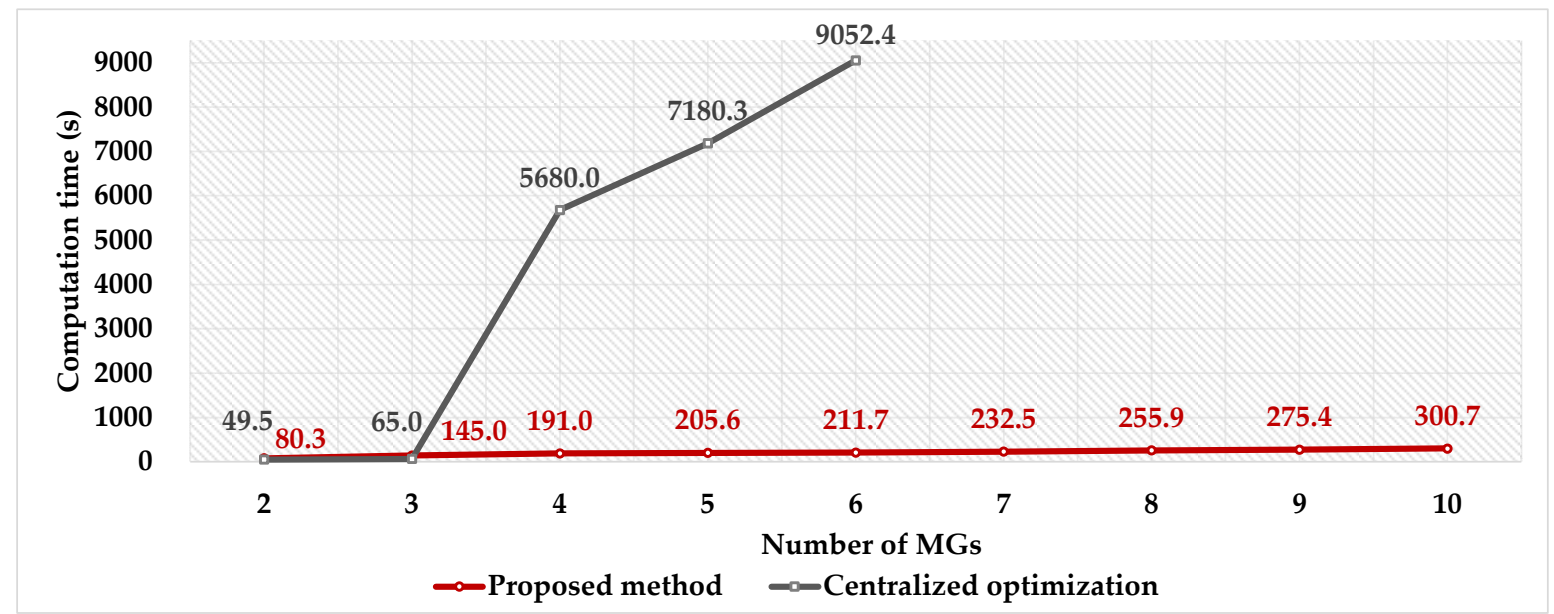

Figure 12. Computation time of the two power sharing scheduling methods according to the number of MGs.

\subsection{Effect of Power Sharing on the System Loss}

If the power is exchanged via the utility distribution network, it may increase the system loss. Therefore, the effect of ESS operation and power sharing on the power loss of the system is analyzed. In this study, a simplified Kron's loss formula is used, as follows [18]:

$$
P_{\text {loss }, \text { sys }}(t)=\sum_{i=1}^{N_{M G}} \alpha_{i} P_{\text {net flow }, i}^{2}(t),
$$


where, $P_{\text {loss,sys }}(t)$ is the system total loss at time $t, \alpha_{i}$ is a loss coefficient of MG $i$, and $P_{\text {net flow }, i}(t)$ is the net flow at the PCC of MG $i$ at time $t$. Firstly, we define the base value of the loss coefficient $\left(\alpha_{b}\right)$ that satisfies the following equation:

$$
P_{\text {loss,sys }}^{a v g}=\alpha_{b}\left(P_{\text {net flow, sys }}^{a v g}\right)^{2},
$$

where, $P_{\text {loss, sys }}^{a v g}$ is the average system loss of a day and $P_{\text {net flow sys }}^{a v g}$ is the average value of the sum of net flows of all MGs. If the system average loss is $l \%$ of the system average net flow $\left(P_{\text {net flow sys }}^{a v g}\right), \alpha_{b}$ can be calculated from Equations (26) and (27) as follows:

$$
\alpha_{b}=N_{M G} \frac{l}{100 P_{\text {net flow,sys }}^{a v g}} .
$$

When $l=5 \%, \alpha_{b}$ was determined to be 0.00025 for the 5-MG system. Since the contribution of each MG to the system loss depends on many factors, including the location and line resistance, we considered three different cases, as summarized in Table 5. In case 1, it was assumed that all MGs have the same loss coefficient value. In case 2, MGs 1 and 4, which send power to other MGs, have higher coefficient values, while MGs 2, 3, and 5, which receive power, have lower values. Case 3 assumes the opposite situation.

Table 5. Three analysis cases with different loss coefficient values.

\begin{tabular}{cccccc}
\hline Case No. & MG1 & MG2 & MG3 & MG4 & MG5 \\
\hline case 1 & $\alpha_{b}$ & $\alpha_{b}$ & $\alpha_{b}$ & $\alpha_{b}$ & $\alpha_{b}$ \\
case 2 & $1.6 \alpha_{b}$ & $0.6 \alpha_{b}$ & $0.6 \alpha_{b}$ & $1.6 \alpha_{b}$ & $0.6 \alpha_{b}$ \\
case 3 & $0.4 \alpha_{b}$ & $1.4 \alpha_{b}$ & $1.4 \alpha_{b}$ & $0.4 \alpha_{b}$ & $1.4 \alpha_{b}$ \\
\hline
\end{tabular}

Figure 13 summarizes the total system loss for the three cases according to three different operation scenarios. It was observed that the loss was increased when the ESSs were used, regardless of whether or not power was shared, in all three cases. Therefore, it cannot be said that utilizing the ESS for power sharing increases the loss more than the use of the ESS for other purposes. The increase in loss is mainly due to the efficiency loss of the ESS during the charge and discharge process. In other words, since the charging energy is greater than the discharging energy, as presented in Equation (22), operating the ESS increases the total net flow of an MG and thus increases the loss. In the three cases, the increase in loss after power sharing was $0.22 \%, 0.31 \%$, and $0.12 \%$, respectively. The loss increased the most in case 2, in which the MGs sending power to other MGs had higher loss coefficient values.

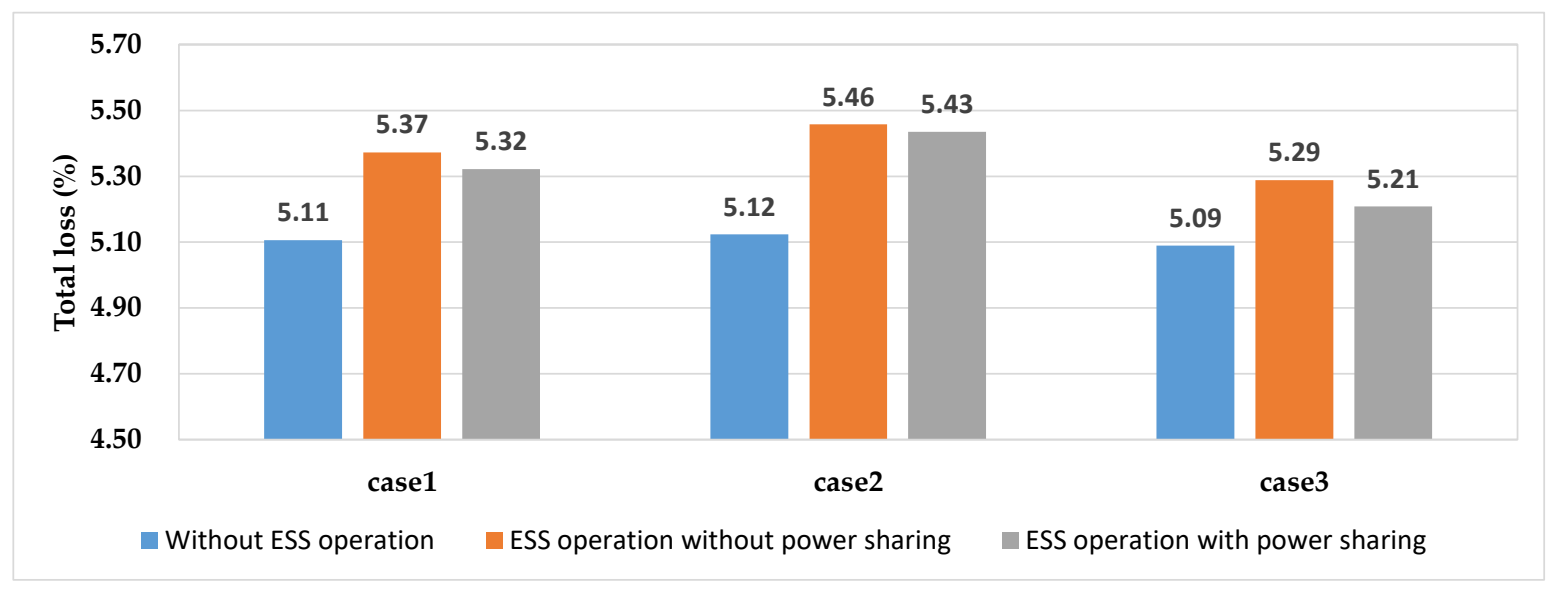

Figure 13. Total system loss according to the loss coefficient values and ESS operation scenarios. 


\subsection{Effect of System Loss on the Power Sharing Schedule}

This section discusses how power sharing schedules are affected when the loss is considered in calculating the operating cost of MGs. If the cost of system loss is included, the objective function is modified as follows:

$$
\min _{T}=\sum_{i=1}^{N_{M G}}\left\{C_{M G, i}+\sum_{t=1}^{T} C_{\text {loss }}(t) \alpha_{i} P_{\text {net flow }, i}(t)^{2}\right\},
$$

where, $C_{\text {loss }}(t)$ is the price of the loss at time $t$, and it is assumed that the price is the same as the TOU rate of MGs 1-4. To analyze the effect of loss, three cases with different average system loss values (l) were simulated: $l=2 \%, l=5 \%$, and $l=15 \%$. In this part, the loss coefficient of case 2 in Table 5 was used because the increase in loss due to the ESS operation was the most significant with this coefficient value.

Table 6 summarizes the operating cost of MGs according to different power sharing schedules. The original schedule refers to the power sharing schedule without considering the loss cost, i.e., the schedule shown in Figure 8. The modified schedule refers to the power sharing schedule calculated by using the modified objective function, including the loss cost. The total operating cost consists of two terms, i.e., the cost calculated by Equation (9) and the loss cost. When $l=2 \%$, the first cost term of the two schedules was the same, but the loss cost was decreased in the modified schedule. In the cases where $l$ was increased to $5 \%$ and $15 \%$, the total cost was reduced by modifying the schedule to further reduce the loss cost, despite a slight increase in the other cost term.

Table 6. Operating cost of MGs according to different power sharing schedules.

\begin{tabular}{ccccccc}
\hline \multirow{2}{*}{$\begin{array}{c}\text { Average } \\
\text { Loss }\end{array}$} & \multicolumn{4}{c}{ Original Schedule } & \multicolumn{3}{c}{ Modified Schedule } \\
& Except Loss & Loss Cost & Total & Except Loss & Loss Cost & Total \\
\hline$l=2 \%$ & $1,468,851.1$ & $31,107.3$ & $1,499,958.5$ & $1,468,851.1$ & $30,545.0$ & $1,499,396.2$ \\
$l=5 \%$ & $1,468,851.1$ & $77,768.4$ & $1,546,619.5$ & $1,469,236.2$ & $75,486.6$ & $1,544,722.7$ \\
$l=15 \%$ & $1,468,851.1$ & $233,305.1$ & $1,702,156.2$ & $1,469,672.5$ & $226,014.6$ & $1,695,687.1$ \\
\hline
\end{tabular}

For a more detailed analysis, Figure 14 shows the power sharing schedules determined by taking into account the loss cost. The impact of loss on the power sharing schedule can be summarized as follows. First, power exchange to reduce the energy cost decreased as the system average loss increased. The amount of power from MGs 1 and 4 to MG 5 at 3:00 decreased from $180 \mathrm{kWh}$ to $100 \mathrm{kWh}$ and $40 \mathrm{kWh}$, respectively, as $l$ increased from $2 \%$ to $5 \%$ and $15 \%$. This is because the increase in the lost cost of the sending MGs outweighs the energy cost saving as the amount of power sharing increases. Second, power exchange may occur to reduce the loss cost, especially when the loss price is high, such as at 10:00, 11:00, and 13:00-16:00 in this scenario. If an individual MG operator is able to estimate the loss, these power sharing schedules may already be reflected in determining the ESS operation schedule. However, if an individual MG operator cannot estimate the loss, a coordinated operation by the MG aggregator can help individual MGs change the ESS operation schedule to contribute to reducing loss costs. Third, even if the loss cost is considered, power sharing schedules between 17:00 and 20:00 to reduce the demand charge were almost not changed. This is evident because the penalty of exceeding the contract demand is much larger than the loss cost savings. 


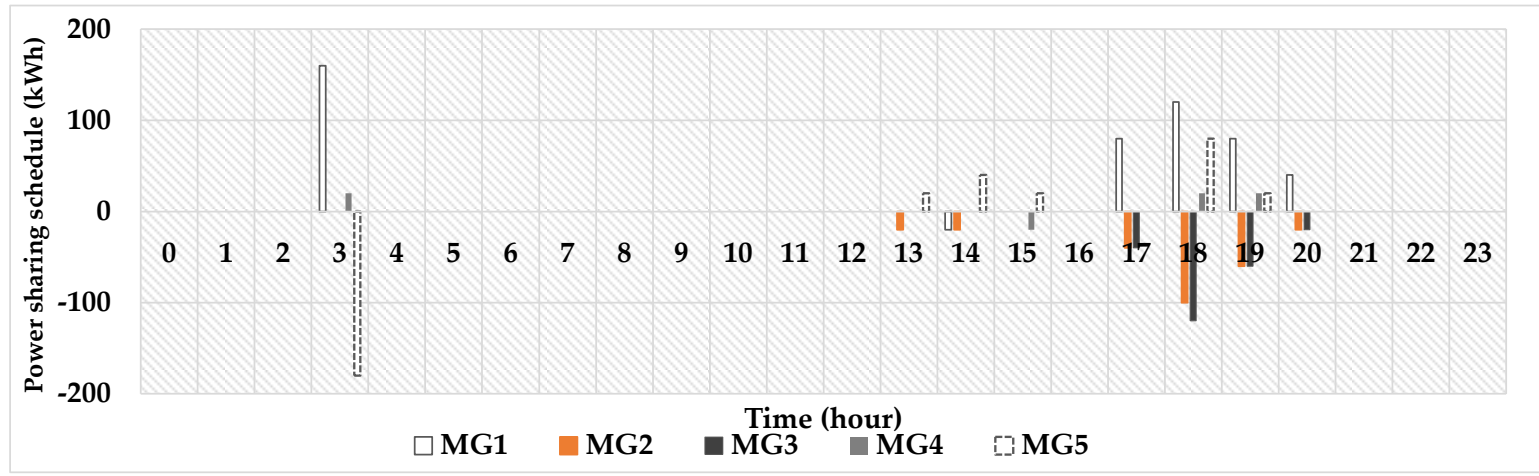

(a)

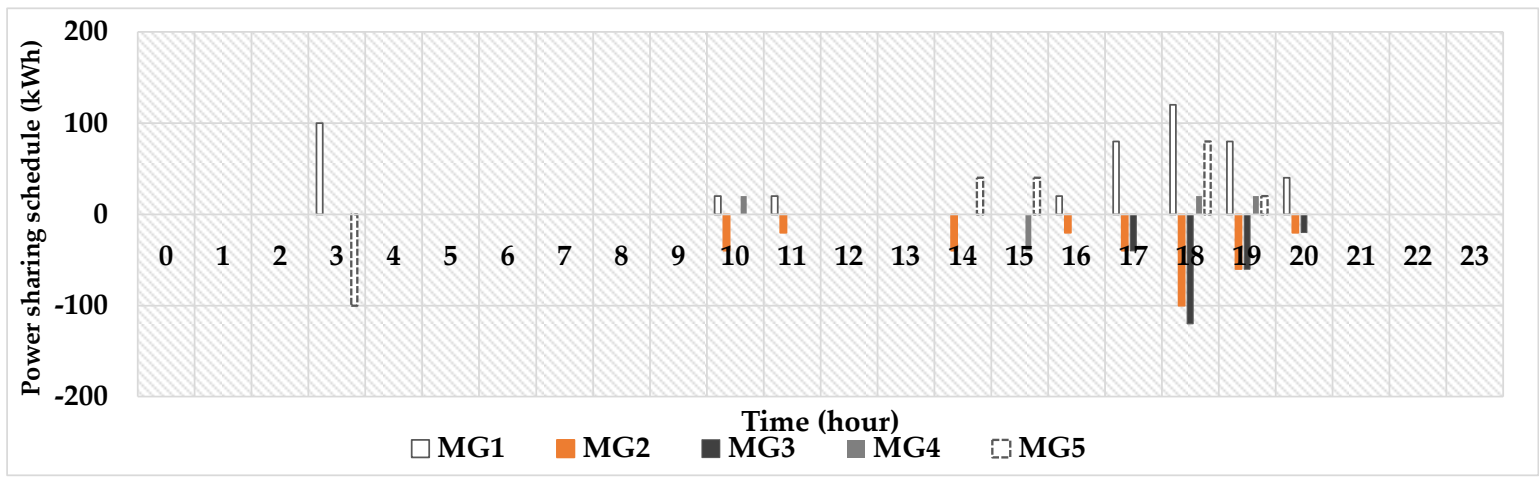

(b)

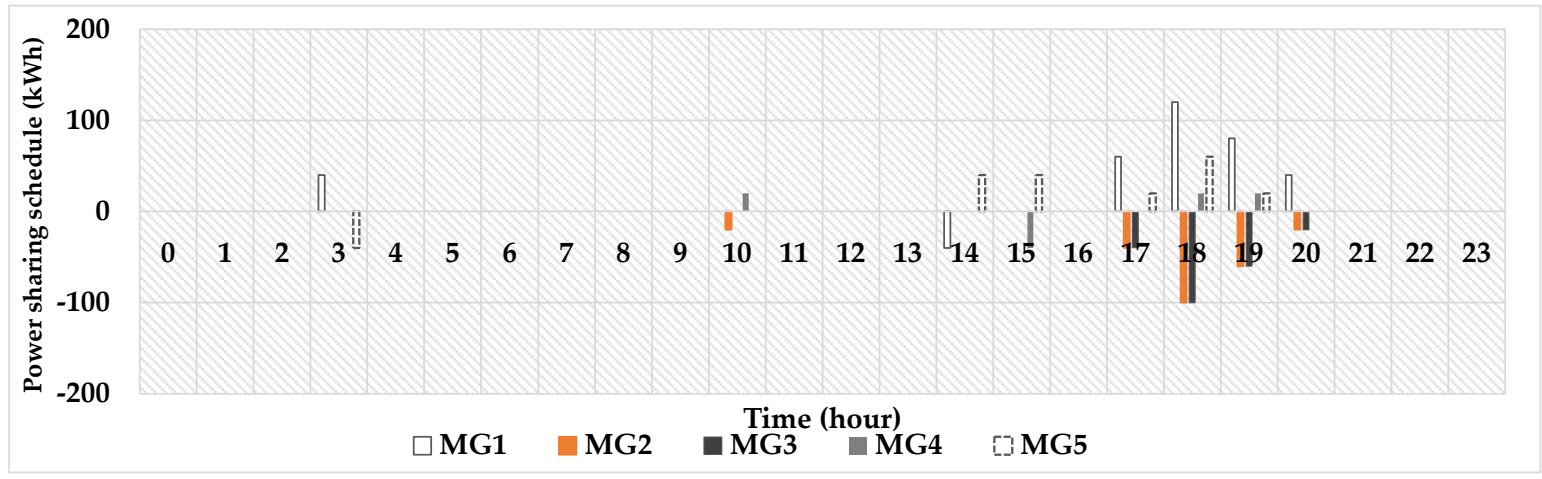

(c)

Figure 14. Power sharing schedule considering the loss cost: (a) $l=2 \%$, (b) $l=5 \%$, and (c) $l=15 \%$.

\section{Conclusions}

In this paper, a power sharing scheme for multiple microgrids that is advantageous to both MG owners and utility has been proposed. In this scheme, the MG that sends power is forced to reduce its net flow by as much power as it sends to other MGs, by discharging the power from the ESS. Two different methods for power sharing scheduling, which is performed by the MG aggregator, were formulated. Firstly, an MILP-based centralized approach, which is an extension of the single MG ESS scheduling problem, was presented. Although this approach can provide the optimal power sharing solution, it was found that the method lacks practical feasibility, due to the large calculation burden. To overcome this shortcoming, a new scheduling algorithm was proposed by assuming that MGs share power in multiples of a unit amount. In this approach, the optimal power sharing schedule was determined by iteratively finding the best two MG pairs that exchange the unit amount of power. The proposed power sharing scheme and scheduling methods were tested for the 5-MG system with a carefully chosen scenario. The overall operating cost of the five MGs could be reduced by about $40 \%$ 
through the power sharing, which represents a huge benefit for the MG owners. Moreover, the daily peak demand of the MGs was reduced by $9.3 \%$ after the power sharing. This proved that the demand profile of aggregated MGs can be improved by the proposed scheme. The utility can also coordinate with the MG aggregators to reduce the peak demand of the distribution system if the proposed power sharing scheme is realized. A comparison of the two power sharing scheduling methods showed that the proposed iterative pairing-based approach can significantly reduce the calculation time, while the operating cost was slightly increased by less than $1 \%$. When the MGs exchanged power through the utility network, power sharing slightly increased the distribution system loss due to the efficiency loss of the ESS. If the loss was taken into account in calculating the operating cost of MGs, power sharing schedules were modified to reduce the loss cost.

Author Contributions: Conceptualization, S.-J.A. and J.-H.C.; methodology, H.-C.G. and S.-J.A.; software, H.-C.G. and S.-Y.Y.; validation, J.-H.C. and S.-Y.Y.; writing-original draft preparation, H.-C.G; writing-review and editing, S.-J.A.; supervision, S.-J.A.; project administration, J.-H.C. and S.-Y.Y. All authors have read and agreed to the published version of the manuscript.

Funding: This research was supported by Korea Electric Power Corporation (Grant number: R18XA04) and the KEPCO Research Institute grant funded by Korea Electric Power Corporation (R16DA11).

Conflicts of Interest: The authors declare no conflict of interest.

\section{References}

1. Wang, J.; Wang, Q.; Zhou, N.; Chi, Y. A Novel Electricity Transaction Mode of Microgrids Based on Blockchain and Continuous Double Auction. Energies 2017, 10, 1971. [CrossRef]

2. Lin, W.M.; Tu, C.S.; Tsai, M.T. Energy Management Strategy for Microgrids by Using Enhanced Bee Colony Optimization. Energies 2016, 9, 5. [CrossRef]

3. Plewnia, F. The Energy System and the Sharing Economy: Interfaces and Overlaps and What to Learn from Them. Energies 2019, 12, 339. [CrossRef]

4. Kalathil, D.; Wu, C.; Poola, K.; Varaiya, P. The Sharing Economy for the Electricity Storage. IEEE Trans. Smart Grid 2019, 10, 556-567. [CrossRef]

5. Zhou, J.; Zhang, J.; Cai, X.; Shi, G.; Wang, J.; Zang, J. Design and Analysis of Flexible Multi-Microgrid Interconnection Scheme for Mitigating Power Fluctuation and Optimizing Storage Capacity. Energies 2019, 12, 2132. [CrossRef]

6. Han, Y.; Li, H.; Shen, P.; Coelho, A.A.E.; Guerrero, M.J. Review of Active and Reactive Power Sharing Strategies in Hierarchical Controlled Microgrids. IEEE Trans. Power Electron. 2016, 32, 2427-2451. [CrossRef]

7. Shamsi, P.; Xie, H.Q.; Longe, A.; Joo, J.Y. Economic Dispatch for an Agent-Based Community Microgrid. IEEE Trans. Smart Grid 2016, 7, 2317-2324. [CrossRef]

8. Kim, B.; Bae, S.H.; Kim, H.S. Optimal Energy Scheduling and Transaction Mechanism for Multiple Microgrids. Energies 2017, 10, 566.

9. Wang, H.; Huang, J.W. Incentivizing Energy Trading for Interconnected Microgrids. IEEE Trans. Smart Grid 2018, 9, 2647-2657. [CrossRef]

10. Taniguchi, T.; Kawasaki, K.; Fukui, Y.; Takata, T.; Yano, S. Automated Linear Function Submission-Based Double Auction as Bottom-up Real Time Pricing in a Regional Prosumers' Electricity Network. Energies 2015, 8, 7381-7406. [CrossRef]

11. Song, N.O.; Lee, J.H.; Kim, H.M.; Im, T.H.; Lee, J.Y. Optimal Energy Management of Multi-Microgrids with Sequentially Coordinated Operations. Energies 2015, 8, 8371-8390. [CrossRef]

12. Lee, W.P.; Choi, J.Y.; Won, D.J. Coordination Strategy for Optimal Scheduling of Multiple Microgrids Based on Hierarchical System. Energies 2017, 10, 1336. [CrossRef]

13. Yang, X.; He, H.; Zhang, Y.; Chen, Y.; Weng, G. Interactive Energy Management for Enhancing Power Balances in Multi-Microgrids. IEEE Trans. Smart Grid 2019, 10, 6055-6069. [CrossRef]

14. Liu, N.; Wang, J. Energy Sharing for Interconnected Microgrids with a Battery Storage System and Renwable Energy Sources Based on the Alternating Direction Method of Multipliers. Appl. Sci. 2018, 8, 1. [CrossRef] 
15. Gao, H.C.; Choi, J.H.; Yun, S.Y.; Lee, H.J.; Ahn, S.J. Optimal Scheduling and Real Time Control Schemes of Battery Energy Storage System for Microgrids Considering Contract Demand and Forecast Uncertainty. Energies 2018, 11, 1371. [CrossRef]

16. Gerard, H.; Rivero, E.; Six, D. Basic Schemes for TSO-DSO Coordination and Ancillary Services Provision, SmartNet Projcet Report. Available online: http://smartnet-project.eu/wp-content/uploads/2016/12/D1.3_ 20161202_V1.0.pdf (accessed on 10 March 2020).

17. Website of KEPCO. Available online: http://cyber.kepco.co.kr/ckepco/front/jsp/CY/E/E/CYEEHP00204.jsp (accessed on 20 October 2019).

18. Saadat, H. Power System Analysis, 2nd ed.; Mc Graw Hill: New York, NY, USA, 2004.

(C) 2020 by the authors. Licensee MDPI, Basel, Switzerland. This article is an open access article distributed under the terms and conditions of the Creative Commons Attribution (CC BY) license (http://creativecommons.org/licenses/by/4.0/). 